\title{
Symmetry and $p$-Stability
}

\author{
Srihari Govindan*, Arndt von Schemde ${ }^{\dagger}$, and \\ Bernhard von Stengel $^{\dagger}$
}

\author{
19 November 2003 \\ CDAM Research Report LSE-CDAM-2003-20
}

\begin{abstract}
A symmetry of a game is a permutation of the player set and their strategy sets that leaves the payoff functions invariant. In this paper we introduce and discuss two relatively mild symmetry properties for set-valued solution concepts (that are equivalent when the solution concepts are single-valued) and show using examples that stable sets satisfy neither version. These examples also show that for every integer $q$, there exists a game with an equilibrium component of index $q$.
\end{abstract}

\section{Introduction}

A symmetry of a game is a permutation of the player set and their strategy sets that leaves the payoff functions invariant. Nash (1951) proved that every finite game has an equilibrium point that is invariant under all the symmetries of the game. This result, and its ready extension to a wider class of games, has proved to be a very useful property. Indeed, in many economic applications (for e.g., the theory of auctions) there is a natural symmetry among the players; and, analyses of these games focus on their symmetric equilibria. Since the 1970s, a steady stream of refinements have been proposed, with little or no attention paid to their symmetry properties. (Cf. van Damme, 1991, for a survey of the refinements literature.) In this paper, we examine the implications of the symmetry axiom for refinements, especially $p$-stable sets as defined by Mertens $(1989,1991)$.

For singleton solution concepts, the formulation of a symmetry axiom is straightforward: we require the existence of a solution that is invariant under all the symmetries of the game. It is very easily verified then that Nash's result extends to (all the different notions of) perfect equilibria, proper equilibria, and sequential equilibria. In fact, persistent equilibrium seems to be the only single-valued solution concept that does not satisfy the

\footnotetext{
*Department of Economics, The University of Iowa, Iowa City, IA 52242-1000, USA. email: srihari-govindan@uiowa.edu

$\dagger$ Department of Mathematics, London School of Economics, Houghton St, London WC2A 2AE, United Kingdom. email: A.von-Schemde@1se.ac.uk, stengel@nash.lse.ac.uk
} 
symmetry axiom: consider the battle of sexes game; its unique symmetric equilibrium is the mixed equilibrium, which is not persistent.

Since stability is a set-valued solution concept, there is not a unique formulation of the symmetry axiom. In Section 2 we provide two, relatively mild, symmetry axioms for stability (that are equivalent for single-valued solution concepts) and show in Section 4 that $p$-stable sets fail to satisfy either of these two axioms. What is not clear to us, at present, is whether stability as defined by Hillas (see Hillas, 1990, and Hillas et al, 2001) violates these axioms, too.

One by-product of our analysis is that the examples we use to prove our result show that for any integer $q$, there exists a game with an equilibrium component of index $q$. While the example for the case $q=0$ is sufficient for proving our main result, we present the entire class of examples, since they are of interest per se.

\section{The Symmetry Axiom}

Let $G$ be a finite normal form game with player set $N$. For each player $n$, let $S_{n}$ (resp., $\left.\Sigma_{n}\right)$ be his pure (resp., mixed) strategy set; and let $\pi_{n}: S \rightarrow \mathbb{R}$ be his payoff function, where $S=\Pi_{n} S_{n}$. We will denote still by $\pi_{n}$ the extension of player $n$ 's payoff function to the set $\Sigma=\Pi_{n} \Sigma_{n}$ of mixed strategy profiles. Finally, let $\vee_{n} S_{n}=\cup_{n}\left(\{n\} \times S_{n}\right)$ be the set sum of the pure strategy sets. The following definition of a symmetry of a game is due to Nash (1951).

Definition 2.1 A symmetry of a game $G$ is a permutation $\phi$ of $\vee_{n} S_{n}$ such that

(a) For each player $n, \phi\left(\{n\} \times S_{n}\right)=\{m\} \times S_{m}$ for some player $m$.

(b) Let $\psi$ and $\varphi$ be, resp., the permutations of $N$ and $S$ that are induced by $\phi$; then, for all $n \in N$, and $s \in S, \pi_{n}(s)=\pi_{\psi(n)}(\varphi(s))$.

The permutation $\varphi$ of $S$ in the definition above extends in the obvious way to a permutation on $\Sigma$, which, too, leaves the payoff functions invariant. Since a symmetry of $G$ is completely specified by the induced permutation of $\Sigma$, we will talk about symmetries only in terms of the $\varphi$ 's. We will say that a subset $\Sigma^{*}$ of $\Sigma$ is invariant under a symmetry $\varphi$ if $\varphi\left(\Sigma^{*}\right)=\Sigma^{*}$. A subset $\Sigma^{*}$ is symmetric if it is invariant under all symmetries of the game.

In formulating a symmetry axiom, we could ask for a solution to be invariant under all symmetries or just the existence of an invariant solution for each symmetry. The former notion is really strong and intuitively appealing. On the other hand, if a game admits many different symmetries, it is conceivable that the equilibrium that is played might depend on which symmetry the players focus on. Hence, the latter notion of symmetry seems an acceptable formulation, as well; in any case, it is certainly the weaker of the two notions and, as such, will be our focus here. 
Kohlberg and Mertens (1986) argued that certain basic axioms force us to consider setvalued solution concepts. For such concepts, there are at least two different formulations. One could require that, for each symmetry, there exists a solution that contains an invariant equilibrium; or that there exists a solution that is invariant. Both these requirements are equally attractive. If we interpret solution concepts as saying that nothing outside them is a rational outcome, then the first formulation would be reasonable since symmetric equilibria are plausible. The second requirement, on the other hand, is a way of requiring solutions themselves to treat equilibria symmetrically. Therefore, we have the following two versions of the Symmetry Axiom.

Axiom 2.2 For every game $G$, and every symmetry $\varphi$ of $G$, there exists a solution $\Sigma^{*}$ that is invariant under $\varphi$.

Axiom 2.3 For every game $G$, and every symmetry $\varphi$ of $G$, there exists a solution $\Sigma^{*}$ that contains a point $\sigma^{*}$ that is invariant under $\varphi$.

The main result of the paper is the following Proposition, which is proved in Section 4 using the examples constructed in Section 3.

Proposition 2.4 p-stable sets satisfy neither of the two symmetry axioms.

\section{Equilibrium Components with Arbitrary Index}

The index of a component of equilibria of a game is an integer that is computed as the local degree of a map for which the Nash equilibria of the game are the zeros. The index is independent of the particular displacement map used and, for generic bimatrix games, it is the negative of the index defined by Shapley (1974) — cf. Govindan and Wilson (1997b), and, for games with any number of players, Demichelis and Germano (2000). In this section, we will show how games with equilibrium components of arbitrary index can be constructed. We explain these constructions in some detail, as readers might find them unfamiliar; they may also be of use in other contexts.

First, consider a $2 \times 2$ coordination game, say

$$
H^{2}=\left[\begin{array}{cc}
10,10 & 0,0 \\
0,0 & 10,10
\end{array}\right]
$$

(in agreement with the notation in (5) below). This game has two pure strategy equilibria, and one mixed equilibrium where both players play the mixed strategy $\left(\frac{1}{2}, \frac{1}{2}\right)$. The index of any of these equilibria is easily determined by the following two properties, which hold for any game: A pure strategy equilibrium which is strict (that is, all unplayed pure strategies have a payoff that is strictly lower than the equilibrium payoff) has index +1 ; 
and the sum over all equilibria of their indices is +1 . Therefore, the mixed equilibrium in $H^{2}$ has index -1 .

Next, we add an outside option called Out to the set of pure strategies of player 1, say, giving the game

$$
G^{-}=\left[\begin{array}{cc}
10,10 & 0,0 \\
0,0 & 10,10 \\
9,9 & 9,9
\end{array}\right]
$$

An outside option (which we may add for one or both players) can be thought of as an initial move that a player can make which terminates further play, and gives a constant payoff to both players. If the player has not chosen his outside option, the original game is played. The outside option payoff above is 9 for both players. This has the effect that an equilibrium of the original game with payoff less than 9 disappears, in this case the mixed strategy equilibrium. Geometrically, one can consider the "upper envelope", that is, the maximum, of the expected payoffs for the pure strategies of player 1 as functions of the mixed strategy played by player 2 . Any equilibrium strategy of player 2 , together with its payoff to player 1, is on that upper envelope. The outside option gives an additional constant function that "cuts off" any former equilibrium payoffs below it.

In the game $G^{-}$, the original pure strategy equilibria of $H^{2}$ are unaffected, and continue to have index +1 . Any such equilibrium, as long as it remains strict after introducing the outside option, keeps its index, as the index of a strict equilibrium can be defined in terms of the payoff sub-matrices corresponding to the pure best responses (cf. Shapley, 1974). The mixed strategy equilibrium of $H^{2}$ is absorbed into an equilibrium component where player 1 plays his last strategy Out. The original mixed equilibrium strategy $\left(\frac{1}{2}, \frac{1}{2}\right)$ of player 2 is part of the outside option component, which is given by the set of mixed strategies of player 2 so that $O u t$ is a best response. In $G^{-}$above, it is easy to see that these are all mixed strategies of player 2 where each pure strategy has probability at most $9 / 10$. In general, the outside option component is defined by a set of linear inequalities, one for each pure strategy of the player who plays Out.

Let $\gamma$ be some game with an outside option. We will denote the outside option equilibrium component of the game $\gamma$ by $\mathcal{C}(\gamma)$. In (1), the index of $C\left(G^{-}\right)$is -1 , which is simply the sum of the indices all equilibria of the original game $H^{2}$ that have been obsorbed into the outside option component, because the sum of all indices is +1 . Technically, the index of an equilibrium component can be defined as the sum of the indices of equilibria near the component when the payoffs are perturbed generically; this sum does not depend on the perturbation.

It is well known that the best response structure of a bimatrix game remains unchanged when adding a constant to any column of the payoffs to the row player, or a constant to a row of the column player's payoffs. This will allow us to cut off pure strategy equilibria rather than mixed equilibria by using an outside option. We start with a $2 \times 2$ coordination game with payoffs 1,1 on and 0,0 off the main diagnal, and add the constant 12 to the first column of player 1 and row of player 2 , and 7 to the second column respectively row. 
The resulting game $H$ and a corresponding outside option game $G$ are given by

$$
H=\left[\begin{array}{cc}
13,13 & 7,12 \\
12,7 & 8,8
\end{array}\right], \quad G=\left[\begin{array}{cc}
13,13 & 7,12 \\
12,7 & 8,8 \\
9,9 & 9,9
\end{array}\right] .
$$

The game $H$ has two pure equilibria with payoffs 13,13 and 8,8, respectively, and one mixed equilibrium where both play $\left(\frac{1}{2}, \frac{1}{2}\right)$ with payoffs 10,10 . The outside option with payoff 9 cuts off the pure strategy equilibrium with payoffs 8,8 but leaves the other equilibria intact. Consequently, $C(G)$ has index +1 .

Next, we "destroy" the pure strategy equilibrium in $G$ by adding another column to the game. Consider the games

$$
H^{\prime}=\left[\begin{array}{ccc}
13,13 & 7,12 & 1,14 \\
12,7 & 8,8 & 2,1
\end{array}\right], \quad G^{\prime}=\left[\begin{array}{ccc}
13,13 & 7,12 & 1,14 \\
12,7 & 8,8 & 2,1 \\
9,9 & 9,9 & 9,9
\end{array}\right] .
$$

Compared to $H$, the pure strategy equilibrium with payoffs 13,13 is no longer present in $H^{\prime}$. It is replaced by another, mixed equilibrium where player 1 plays $\left(\frac{6}{7}, \frac{1}{7}\right)$ and player 2 plays $\left(\frac{1}{2}, 0, \frac{1}{2}\right)$, with payoffs 7 to player 1 and $85 / 7$ to player 2 . This new mixed equilibrium has index +1 . Since the payoff to player 1 in that equilibrium is less than the outside option payoff 9, that equilibrium disappears in $G^{\prime}$. Consequently, $C\left(G^{\prime}\right)$ has index +2 , because the only equilibrium that is not cut off has index -1 .

Finally, we consider the following game $H^{-}$which is a symmetrized version of $H^{\prime}$ :

$$
H^{-}=\left[\begin{array}{ccc}
13,13 & 7,12 & 1,14 \\
12,7 & 8,8 & 2,1 \\
14,1 & 1,2 & 1,1
\end{array}\right]
$$

In this game, the mixed strategy equilibrium where both players play $\left(\frac{1}{2}, \frac{1}{2}, 0\right)$ is the equilibrium with the highest payoff, yielding 10 for both players. This equilibrium has index -1 . The other equilibria are as follows: The mixed strategy $\left(\frac{1}{2}, 0, \frac{1}{2}\right)$ of player 2 , which together with $\left(\frac{6}{7}, \frac{1}{7}\right)$ of player 1 forms an equilibrium of $H^{\prime}$, is no longer part of an equilibrium as the third strategy of player 1 in $H^{-}$gives a higher payoff. By playing that strategy as well, we obtain a completely mixed equilibrium where both players play $\left(\frac{1}{2}, \frac{1}{12}, \frac{5}{12}\right)$, with resulting payoff $15 / 2$ to both players. This equilibrium has index +1 , as has the pure strategy equilibrium with payoffs 8,8 . There are no other equilibria of $H^{-}$.

We use $H^{-}$for constructing components with arbitrarily high positive index. For $k \geqslant 1$, let $H^{-k}$ be the game consisting of $k$ copies of the game $H^{-}$on the diagonal and zeros everywhere else, that is,

$$
H^{-k}=\underbrace{\left[\begin{array}{cccc}
H^{-} & 0,0 & \cdots & 0,0 \\
0,0 & H^{-} & & 0,0 \\
\vdots & & \ddots & \vdots \\
0,0 & 0,0 & \cdots & H^{-}
\end{array}\right]}_{k \text { copies }} .
$$


Each player has $3 k$ strategies in $H^{-k}$. For any nonempty set of the $k$ copies of $H^{-}$, and any equilibrium in such a copy, one obtains an additional equilibrium of $H^{-k}$ by suitable probability weights assigned to the copies. All such mixtures involving more than one copy, however, give payoffs less than 8 . There are no other equilibria of $H^{-k}$ as the payoffs in a copy of $H^{-}$are all positive, and the other payoffs are zero.

The superscript in $H^{-k}$ indicates the sum of indices of those equilibria that are not cut off by adding a suitable outside option. To preserve symmetry between players, we give both players an outside option Out as an additional pure strategy, which gives the game

$$
G^{k+1}=\left[\begin{array}{ccc}
H^{-k} & 9,9 \\
& & \vdots \\
9,9 & \cdots & 9,9
\end{array}\right] .
$$

The game $G^{k+1}$ has $k+1$ equilibrium components: the $k$ mixed strategy equilibria where both players play strategies 1 and 2 in one copy of $H^{-}$with probability $\frac{1}{2}$ (yielding a payoff of 10 for both), and the equilibrium component in which at least one player chooses the last strategy, the outside option Out. That component $\mathcal{C}\left(G^{k+1}\right)$ is given by those strategy pairs where at least one player plays Out, and the other player playing such that Out is a best response. The unique symmetric strategy pair in that component is $($ Out, Out $)$. All isolated equilibria have index -1 . Since the indices of all equilibrium components have to add up to one, the outside option equilibrium component $\mathcal{C}\left(G^{k+1}\right)$ has index $k+1$, which we chose as a superscript for $G$ in (4). Therefore, for each positive integer $q$, the game $G^{q}$ in (4) has a component with index $q$; this includes the trivial case $q=1, k=0$, which is a $1 \times 1$ game.

A similar, simpler construction gives equilibrium components with arbitrary negative index. For $k \geqslant 2$, let $H^{k}$ be the following $k \times k$ game:

$$
H^{k}=\underbrace{\left[\begin{array}{cccc}
10,10 & 0,0 & \cdots & 0,0 \\
0,0 & 10,10 & & 0,0 \\
\vdots & & \ddots & \vdots \\
0,0 & 0,0 & \cdots & 10,10
\end{array}\right]}_{k \text { columns }}
$$

Just as (4) is obtained from (3), we add outside options for both players, and obtain

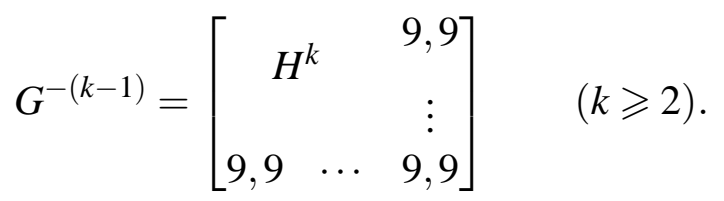

The equilibria of game $G^{-(k-1)}$ are the $k$ pure strategy equilibria of the coordination game, yielding a payoff of 10 for both players, and the outside option equilibrium component $\mathcal{C}\left(G^{-(k-1)}\right)$. Since pure strategy equilibria have index +1 , it follows that $\mathcal{C}\left(G^{-(k-1)}\right)$ 
has index $-(k-1)$. Hence, for each negative integer $q$, there exists a game that has an equilibrium component with index $q$. The case $k=1$ gives an equilibrium with index 0 , but this game is too symmetric for our purpose, which is why we require $k \geqslant 2$ in (6).

In order to show that 0 -stable sets violate the symmetry axioms, we construct a game whose only symmetric equilibrium component has index 0 . We are doing this by combining the games $H^{k}$ and $H^{-(k-1)}$ to a new game by placing them on the diagonal, and adding outside options as before. For our purpose, $k=3$ is sufficient. Thus, let $G^{0}$ be the following $10 \times 10$ game:

$$
G^{0}=\left[\begin{array}{ccc}
H^{3} & 0 & 9,9 \\
0 & H^{-2} & \vdots \\
9,9 & \cdots & 9,9
\end{array}\right] .
$$

As argued after (3), the only equilibria in $G^{0}$ that are not cut off are those with payoffs 10,10 in $H^{3}$ or $H^{-2}$. Thus, by a counting argument, the outside option equilibrium component $\mathcal{C}\left(G^{0}\right)$ has index 0 . Our constructions prove the following proposition.

Proposition 3.1 For each integer q, there exists a (bimatrix) game that has a component of equilibria with index $q$.

Let now $q \neq 1,2$ be some integer and consider the game $G^{q}$ as defined in (4), (6), or (7), respectively. Then the component $\mathcal{C}\left(G^{q}\right)$, which by construction has index $q$, is the unique component of equilibria that is symmetric, i.e., invariant under all symmetries of the game, and (Out, Out $)$ is the unique symmetric equilibrium. Moreover, there exists a permutation under which $C\left(G^{q}\right)$ is the unique invariant component and (Out, Out) is the unique invariant equilibrium.

Example 3.2 For the game in (7), the outside option component $\mathcal{C}\left(G^{0}\right)$ (respectively, the equilibrium (Out,Out)) is the only equilibrium component (respectively, equilibrium) that is invariant under the symmetry that leaves the players invariant, but permutes the strategies with the following permutation, written as a product of cycles, assuming the first nine strategies are 1,2,..9:

$$
(123)(47)(58)(69)(\text { Out }) \text {. }
$$

The first part of the permutation has order 3, and permutes the game $H^{3}$. The middle part of the permutation has order 2 , and permutes the game $\mathrm{H}^{-2}$. The last part is the identity on the outside option, having order 1. Hence, the whole permutation has order 6.

The initial examples show that an outside option for only one player would be sufficient to construct an equilibrium component with arbitrary index $q$. However, since we are interested in the symmetry properties of a game, we add outside options for both players. 


\section{Analysis of the Game $G^{q}$}

Before we analyse the stable sets of the games $G^{q}$, we characterize the perfect equilibria of the component $\mathcal{C}\left(G^{q}\right)$. Let $\mathcal{P}\left(G^{q}\right)$ denote the subset of perfect equilibria of $\mathcal{C}\left(G^{q}\right)$.

Lemma 4.1 The subset $\mathcal{P}\left(G^{q}\right)$ of perfect equilibria of $\mathcal{C}\left(G^{q}\right)$ is path-connected.

Proof. For a two-player bimatrix game, an equilibrium is perfect if and only if it is in undominated strategies, i.e., if and only if there exists a completely mixed strategy against which it is a best reply. This already implies that $(O u t, O u t)$ is perfect, since Out is the unique best reply against the uniform strategy of the other player. We will now show that every other perfect equilibrium in $\mathcal{P}\left(G^{q}\right)$ can be path-connected with (Out, Out).

At least one of the two players plays $O u t$ with probability 1 in the component $C\left(G^{q}\right)$. Therefore, and for reasons of symmetry, we can restrict our attention to perfect equilibria of the form $\left(\sigma_{1}\right.$, Out $)$. Now let $\eta$ be a completely mixed strategy against which $\sigma_{1}$ is a best reply. This implies that the strategies used in $\sigma_{1}$ must give a payoff of at least 9 against $\eta$. Let $H$ be the game obtained from $G^{q}$ by deleting the strategy Out for both players, and consider the completely mixed strategy equilibrium, call it $\tau$, of $H$ (which exists, see the comments following (2) and (3) above). This equilibrium gives both players a payoff strictly smaller than 9 . Hence there exists $\lambda \in[0,1)$ such that $\sigma_{1}$ is a best reply against $\lambda \tau+(1-\lambda) \eta$, and the strategies played in $\sigma_{1}$ yield a payoff of 9 . Note that $\tilde{\eta}:=\lambda \tau+(1-\lambda) \eta$ is a completely mixed strategy. But then $\mu \sigma_{1}+(1-\mu)$ Out is a best reply against $\tilde{\eta}$ for all $\mu \in[0,1]$, showing that $\left(\mu \sigma_{1}+(1-\mu)\right.$ Out, Out $) \in \mathcal{P}\left(G^{q}\right)$, which implies that the set $\mathcal{P}\left(G^{q}\right)$ is star-shaped and, hence, path-connected.

The same analysis is possible in a game where only one player has an outside option. This gives the following property of generic outside option equilibrium components:

Corollary 4.2 The set of perfect equilibria in an outside option equilibrium component is path-connected if the payoffs for the equilibria that have been cut off are generic and if the original game has an equilibrium in completely mixed strategies.

Since stable sets are connected sets of perfect equilibria, the following Proposition, in conjunction with the remarks in Example 3.2, proves Proposition 2.4.

Proposition 4.3 Let $p$ be either zero or a prime number greater than 1. Then, $\mathcal{P}\left(G^{q}\right)$ contains a $p$-stable set iff $q \neq 0$ and $p$ does not divide $q$. In particular, $\mathcal{P}\left(G^{0}\right)$ does not contain a p-stable set for any $p$.

Before proving the above Proposition, we review the concept of $p$-stability. Let $\Sigma^{i}$ denote the mixed strategy set of player $i$, and $\Sigma=\Sigma^{1} \times \Sigma^{2}$. Let $m$ be the number of pure strategies of each player. Given a vector $\eta \in \mathbb{R}_{+}^{m} \times \mathbb{R}_{+}^{m}$, one defines a perturbed game $G^{q}(\eta)$ as the game where the strategy sets are the same as in $G^{q}$ but where 
the payoff to player $i$ from a strategy profile $\sigma$ is the payoff he gets under the profile $\left(\left(1+\bar{\eta}^{1}\right)^{-1}\left(\sigma^{1}+\eta^{1}\right),\left(1+\bar{\eta}^{2}\right)^{-1}\left(\sigma^{2}+\eta^{2}\right)\right)$ in $G^{q}$, where for each $j=1,2, \bar{\eta}^{j}=$ $\sum_{k=1}^{m} \eta_{k}^{j}$. For each $0 \leqslant \varepsilon \leqslant 1$, define

$$
P_{\varepsilon}=\left\{\eta \in \mathbb{R}_{+}^{m} \times \mathbb{R}_{+}^{m} \mid \text { for } i=1,2, \bar{\eta}_{i} \leqslant \varepsilon\right\} .
$$

We use $\partial P_{\varepsilon}$ to denote the topological boundary of $P_{\varepsilon}$. Let $N$ the graph of the equilibrium correspondence defined over $P_{1}$, i.e.,

$$
N=\left\{(\eta, \sigma) \in P_{1} \times \Sigma \mid \sigma \text { is an equilibrium of } G^{q}(\eta)\right\},
$$

and let $N_{\varepsilon}$ be defined accordingly. Denote by proj the natural projection from $N$ to $P_{1}$. For each $X \subseteq N$, and $\varepsilon \geqslant 0$, let $\left(X_{\varepsilon}, \partial X_{\varepsilon}\right)=\operatorname{proj}^{-1}\left(P_{\varepsilon}, \partial P_{\varepsilon}\right) \cap X$. In the following definition, we use simplicial homology with coefficients in $\mathbb{Z}_{p}\left(\right.$ where $\left.\mathbb{Z}_{0}=\mathbb{Z}\right)$.

Definition 4.4 The p-stable sets of $G^{q}$ are the Hausdorff limits of the sets $\{\sigma \mid(0, \sigma) \in X\}$ where $X$ varies over those closed, semi-algebraic subsets of $N$ such that:

(a) Connexity: $X_{\varepsilon} \backslash \partial X_{\varepsilon}$ is connected and dense in $X_{\varepsilon}$ for each sufficiently small $\varepsilon>0$.

(b) Essentiality: proj: $H_{*}\left(X_{\varepsilon}, \partial X_{\varepsilon} ; \mathbb{Z}_{p}\right) \rightarrow H_{*}\left(P_{\varepsilon}, \partial P_{\varepsilon} ; \mathbb{Z}_{p}\right)$ is nonzero for some, and then for all sufficiently small, $\varepsilon>0$.

Each of the isolated equilibria of $G^{q}$ is $p$-stable: indeed, since these equilibria are regular, the projection map from a neighbourhood of the equilibrium in $N$ is a homeomorphism onto $P_{\varepsilon}$ for small $\varepsilon$ and thus satisfies the connexity and the essentiality condition.

We will now prove Proposition 4.3. By Govindan and Wilson (2001), if $\mathcal{P}\left(G^{q}\right)$ contains a $p$-stable set, then $\mathcal{P}\left(G^{q}\right)$ is itself $p$-stable. Therefore, it suffices to show that this component is not a $p$-stable set if $q=0$ or $p$ divides $q$.

Choose an open neighbourhood $U$ of $C$ in $\Sigma$ such that its closure does not contain any of the isolated equilibria of $G^{q}$. Govindan and Wilson (2001, Remark 3.3) show that there exists $\varepsilon_{0}>0$ so that the closure $S$ of the set $\left\{(\eta, \sigma) \in N_{\varepsilon_{0}} \backslash \partial N_{\varepsilon_{0}} \mid \sigma \in U\right\}$ satisfies the connexity condition of Definition 4.4. (The formulation in Govindan and Wilson, 2001 , is different from ours in a few inessential aspects. Firstly, for every perturbation $\eta$, they consider the game $G^{q}(\eta /(1-\bar{\eta}))$. Secondly, they consider the graph of the perturbed equilibrium correspondence. Clearly, there is a homeomorphism between the two equilibrium graphs that commutes with the respective projections, so that the connexity property is preserved.) Thus, for every $0<\varepsilon \leqslant \varepsilon_{0}$, the set $S_{\varepsilon} \backslash \partial S_{\varepsilon}$ is connected and dense in $S_{\varepsilon}$, where $S_{\varepsilon}$ is the closure of the set $\left\{(\eta, \sigma) \in N_{\varepsilon} \backslash \partial N_{\varepsilon} \mid \sigma \in U\right\}$. Moreover, $S$ is a closed semi-algebraic subset of $N$ such that $\mathcal{P}\left(G^{q}\right)=\{\sigma \mid(0, \sigma) \in S\}$. For future use, we will also assume that $\varepsilon_{0}$ is chosen to be small enough such that for each $\eta \in P_{\varepsilon_{0}}$, none of its equilibria belongs to the boundary of $U$.

If $S$ contains a set $X$ that satisfies the essentiality condition, then $S$ would satisfy the essentiality condition as well. Therefore, $\mathcal{P}\left(G^{q}\right)$ is $p$-stable iff $S$ satisfies the essentiality requirement. 
Let $A^{i}$ be player $i$ 's payoff matrix in $G^{q}$. Every vector $\left(g^{1}, g^{2}\right)$ in $\Gamma \equiv \mathbb{R}^{m} \times \mathbb{R}^{m}$ defines a perturbed game in which player $i$ 's payoff matrix has $A_{j k}^{i}+g_{j}^{i}$ as its $(j, k)$-entry. Define a map $f: P_{1} \rightarrow \Gamma$ by $f(\eta)=g$ with $g_{j}^{1}=A_{j}^{1} \cdot \eta^{2}$ and $g_{j}^{2}=A_{j}^{2} \cdot \eta^{1}$. Then the game $f(\eta)$ has the same set of equilibria as $G^{q}(\eta)$. Observe that $f$ is a homeomorphism, since $A^{1}$ and $A^{2}$ are nonsingular. For each $0 \leqslant \varepsilon \leqslant \varepsilon_{0}$, let $\left(Q_{\varepsilon}, \partial Q_{\varepsilon}\right)=f\left(P_{\varepsilon}, \partial P_{\varepsilon}\right)$ and let $\left(T_{\varepsilon}, \partial T_{\varepsilon}\right)$ be the image of $\left(S_{\varepsilon}, \partial S_{\varepsilon}\right)$ under the homeomorphism $f \times$ id. It is sufficient to show that $\mathcal{P}\left(G^{q}\right)$ is $p$-stable iff the projection map from $\left(T_{\varepsilon}, \partial T_{\varepsilon}\right)$ to $\left(Q_{\varepsilon}, \partial Q_{\varepsilon}\right)$ is essential for all small $\varepsilon>0$.

Fix $0<\varepsilon \leqslant \varepsilon_{0}$. Since $f$ is a homeomorphism, $\left(Q_{\varepsilon}, \partial Q_{\varepsilon}\right)$ is a $2 m$-ball with boundary. Let $E$ be the graph of the Nash equilibrium correspondence over $\Gamma$. By the definition of the set $S$, we have that $T_{\varepsilon} \backslash \partial T_{\varepsilon}$ equals $E \cap\left(Q_{\varepsilon} \backslash \partial Q_{\varepsilon} \times U\right)$ and is, therefore, open in $E$. It follows readily from the proof of the Kohlberg-Mertens structure theorem (1986, Proposition 3.1) that $E$ is homeomorphic to $\Gamma$; hence, $T_{\varepsilon} \backslash \partial T_{\varepsilon}$, is an open semi-algebraic $2 m$-manifold. Moreover, $T_{\varepsilon} \backslash \partial T_{\varepsilon}$ is connected and dense in $T_{\varepsilon}$ as $S$ satisfies the connexity condition. Consequently, $\left(T_{\varepsilon}, \partial T_{\varepsilon}\right)$ is a pseudomanifold with boundary. (Cf. Munkres, 1984, $\S 63$ Ex. 3.)

By Govindan and Wilson (1997a), the index of $C\left(G^{q}\right)$ can be computed as the local degree of the projection map from a neighbourhood of the component in $E$ to $\Gamma$, for a suitable orientation of $E$. By the choice of $\varepsilon_{0}$, there exists a neighbourhood of $W$ of $\left(Q_{\varepsilon}, \partial Q_{\varepsilon}\right)$ such that no game in $W$ has an equilibrium on the boundary of $U$. Therefore, the projection map from $V:=E \cap(W \times U)$ is proper over $W$. Since the index of the component $\mathcal{C}\left(G^{q}\right)$ is $q$, for every game $g \in W$, the sum of the indices of the components of its equilibria that are contained in $U$ is $q$. Hence, the projection map from $\left(T_{\varepsilon}, \partial T_{\varepsilon}\right)$ to $\left(Q_{\varepsilon}, \partial Q_{\varepsilon}\right)$ has degree $q$. If $q$ is zero, the map has degree zero; and for $q \neq 0$ the map is essential $\bmod p$ iff $p$ does not divide $q$. Thus $\mathcal{P}\left(G^{q}\right)$ is $p$-stable iff $q \neq 0$ and $p$ does not divide $q$, proving Proposition 4.3.

As an immediate consequence of the proof of Proposition 4.3, we obtain the following Corollary.

Corollary 4.5 Let $\mathcal{C}$ be an equilibrium component of index $q$ of a bimatrix game with payoff matrices $A^{1}$ and $A^{2}$ for player 1 and 2 , respectively. If $A^{1}$ and $A^{2}$ have full rank and if $\mathcal{P}(C)$ is connected, then $C$ is $p$-stable if and only if $q \neq 0$ and $p$ does not divide $q$.

Remark 4.6 For every abelian group $M$ and a subgroup $M^{\prime}$, Mertens (1991) defines $\left(M, M^{\prime}\right)$-stability just like p-stability, with the essentiality condition modified as follows: $\operatorname{proj}_{*}\left(H_{*}\left(S_{\varepsilon}, \partial S_{\varepsilon} ; M\right)\right)$, viewed as a subset of $M \approx H_{*}\left(P_{\varepsilon}, \partial P_{\varepsilon} ; M\right)$, is not contained in $M^{\prime}$. The special case of $\left(M, M^{\prime}\right)=\left(\mathbb{Z}_{p}, 0\right)$ corresponds to the p-stable sets. It is readily seen that $\mathcal{P}\left(G^{0}\right)$ does not contain an $\left(M, M^{\prime}\right)$-stable set for any pair $\left(M, M^{\prime}\right)$. Thus all these other variants fail to satisfy either of our symmetry axioms. Moreover, asking for essentiality in homotopy —which is the idea of homotopy stability (see Mertens, 1991, Section 4)_does not yield a positive result either: in the game $G^{0}$, the projection map from $\left(S_{\varepsilon}, \partial S_{\varepsilon}\right)$ to $\left(P_{\varepsilon}, \partial P_{\varepsilon}\right)$ is homotopic to a map to the boundary (as maps between pairs), 
since the projection map has degree zero as a map from a pseudomanifold with boundary to a ball pair.

Remark 4.7 As remarked in the Introduction, we do not know if the set $\mathcal{P}\left(G^{q}\right)$ contains a stable set in the sense of Hillas.

\section{References}

DeMichelis, S., and F. Germano (2000), On the indices of zeros of Nash fields. Journal of Economic Theory 94, 192-217.

Govindan, S. and R. Wilson (1997a), Equivalence and invariance of the index and degree of Nash equilibria. Games and Economic Behavior 21, 56-61.

Govindan, S. and R. Wilson (1997b), Uniqueness of the index for Nash equilibria of two-player games. Economic Theory 10, 541-549.

Govindan, S. and R. Wilson (2001), Maximal stable sets of two-player games. International Journal of Game Theory 30, 557-566.

Hillas, J. (1990), On the definition of the strategic stability of equilibria. Econometrica 58, 13861390.

Hillas, J., M. Jansen, J. Potters, and D. Vermeulen (2001), On the relation among some definitions of strategic stability. Mathematics of Operations Research 26, 611-635.

Kohlberg, E. and J.-F. Mertens (1986), On the strategic stability of equilibria. Econometrica 54, 1003-1037.

Mertens, J.-F. (1989), Stable equilibria-a reformulation, Part I. Mathematics of Operations Research $14,575-625$.

Mertens, J.-F. (1991), Stable equilibria—a reformulation, Part II. Mathematics of Operations Research 16, 694-753.

Munkres, J. (1984), Elements of Algebraic Topology. Addison-Wesley, Boston.

Nash, J. (1951), Non-cooperative games. Annals of Mathematics 54, 286-295.

Shapley, L. S. (1974), A note on the Lemke-Howson algorithm. Mathematical Programming Study 1: Pivoting and Extensions, 175-189.

van Damme, E. (1991), Stability and Perfection of Nash Equilibria. Springer-Verlag, Berlin. 